\title{
Effect of low-intensity pulsed ultrasound on murine calvarial derived periosteal cells
}

\section{BASIC RESEARCH}

\author{
Waimyo Maung, Hidemi Nakata, Motoi Miura, Munemitsu \\ Miyasaka, You-Kyoung Kim, Shohei Kasugai, Shinji Kuroda \\ Oral Implantology and Regenerative Dental Medicine, \\ Tokyo Medical and Dental University, Japan
}

\section{Abstract}

Background: The replacement of lost natural teeth using osseointegrated implants require adequate bone volume permitting their anchorage. Many studies have shown potential advantages of stem-cell based therapies in bone tissue engineering. Periosteal cells have been suggested as a promising source for bone tissue engineering, and lowintensity pulsed ultrasound (LIPUS) has shown successful bone healing of delayed unions, non-unions and simulated fracture healing in animal models and in clinical treatments. Objective: The aim of this study was to investigate the effect of LIPUS on the proliferation and the osteogenic differentiation of murine calvarial derived periosteal cells. Materials and methods: Four-week-old SLC/ICR female mice were euthanized, and then their calvarias were extracted and washed with phosphate-buffered saline (PBS). Six hundreds $\mathrm{mg}$ gelatin was inserted inside a $60 \mathrm{~mm}$ culture dish and immersed in a $6 \mathrm{ml}$ culture medium. The periosteum was derived from the calvarias and put it onto the gelatin. Then the gelatin was inverted upside-down and incubated at $37^{\circ} \mathrm{C}$ with $5 \% \mathrm{CO} 2$. The culture medium was changed every three days. Cells at the passage of three were treated with LIPUS with an intensity of $30 \mathrm{~mW} / \mathrm{cm} 2$, frequency of $3 \mathrm{MHz}$, pulse repetition frequency (PRF) of $1 \mathrm{kHz}$ and exposure time of $20 \mathrm{~min}$ per day up to 28 days. The cell proliferation and viability were analyzed by cell counting kit-8 (CCK-8) assay, PrestoBlue assay. Osteogenic differentiation was determined by alkaline phosphatase (ALP) staining, ALP activity assay, alizarin red staining and RT-real-time-PCR of osteogenic marker genes.

Results: Cell proliferation is significantly accelerated by LIPUS more than that of the control group. Furthermore, more osteogenic differentiation was observed in LIPUS treated groups.

Conclusion and Clinical implications: The results suggested that LIPUS could enhance cell proliferation and osteogenic differentiation of murine calvarial derived periosteal cells and could be part of effective treatment methods for future clinical applications.

\section{Background and Aim}

Background: The replacement of lost natural teeth using osseointegrated implants require adequate bone volume permitting their anchorage. Many studies have shown potential advantages of stem-cell based therapies in bone tissue engineering. Periosteal cells have been suggested as a promising source for bone tissue engineering.

Studies have indicated that the osteoblast differentiation process of periosteum derived cells can be induced by various factors, including soluble molecules, biophysical or chemical signals, and microenvironment. Ultrasound stimulation belongs to a biophysical signal that can be conducted into the body, and results in biochemical events at the cellular level. ${ }^{1}$ LIPUS has shown successful bone healing of delayed unions, non-unions and simulated fracture healing in animal models and in clinical treatments. LIPUS enhances cell proliferation and mineralization of osteoblast cells through HSP 90.

Aim: The aim of this study was to investigate the effect of LIPUS on the proliferation and the osteogenic differentiation of murine calvarial derived periosteal cells.

\section{Methods and Materials}

Isolation of PDCs

- Calvarial bone of 4-week-old female ICR mice were extracted.

- Periosteum was harvested from the bones, and immersed in the following gelatin medium.

- Periosteum derived cells were isolated using $0.6 \mathrm{~g}$ of gelatin (low endotoxin gelatin; Nippi, Japan) with $6 \mathrm{~mL}$ of DMEM (Sigma-Aldrich, USA) supplemented with 10\% FBS (SigmaAldrich) and 1\% penicillin/streptomycin (Sigma-Aldrich).

- Culture medium were changed every three days.

- Cells at the passage of three were used for experiment.
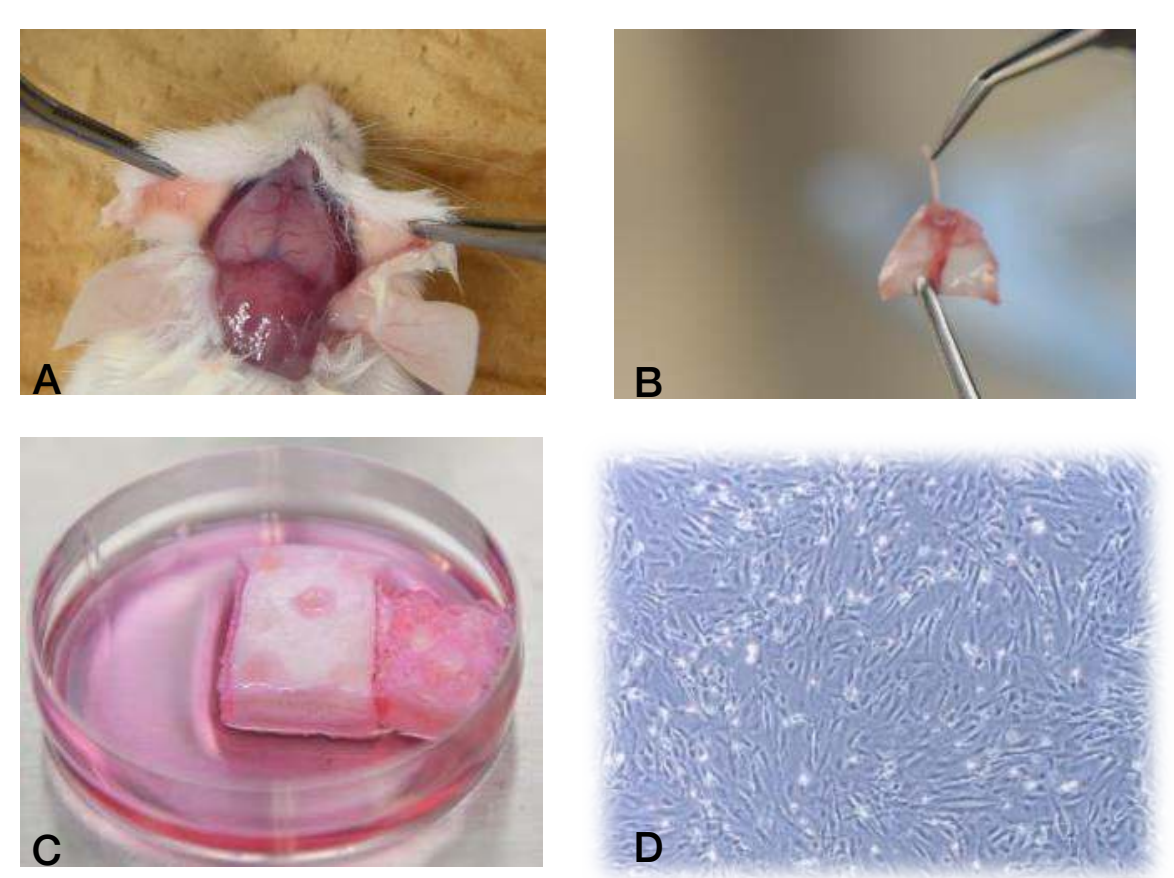

Figure 1. Isolation of murine calvarial derived periosteal cells

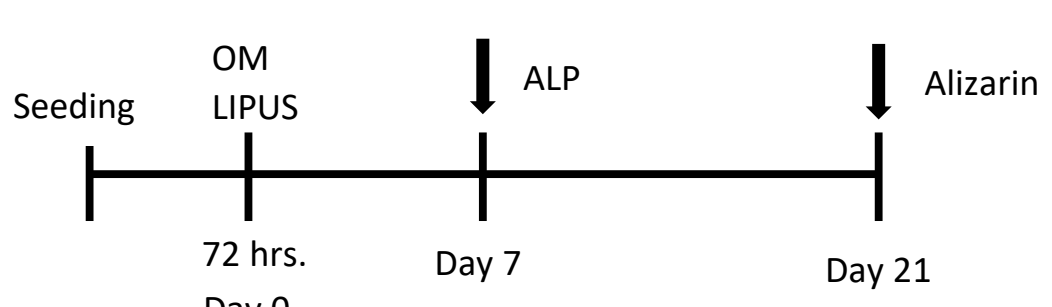

Figure 2. Research time-line. OM = osteogenic medium, LIPUS = low-intensity pulsed ultrasound application

\section{Ultrasound stimulation}

-In all groups, $3 \times 105$ cells were seeded into a 6-well culture plates.

-In L+ groups, cells were exposed to LIPUS stimulation at an intensity of $30 \mathrm{~mW} / \mathrm{cm} 2$, frequency of $3 \mathrm{MHz}$ and pulse repetition frequency (PRF) of $1 \mathrm{kHz}$, duty cycle $20 \%$ (pulse ratio 1:4, SATA) for 20 min per day up to 21 days.
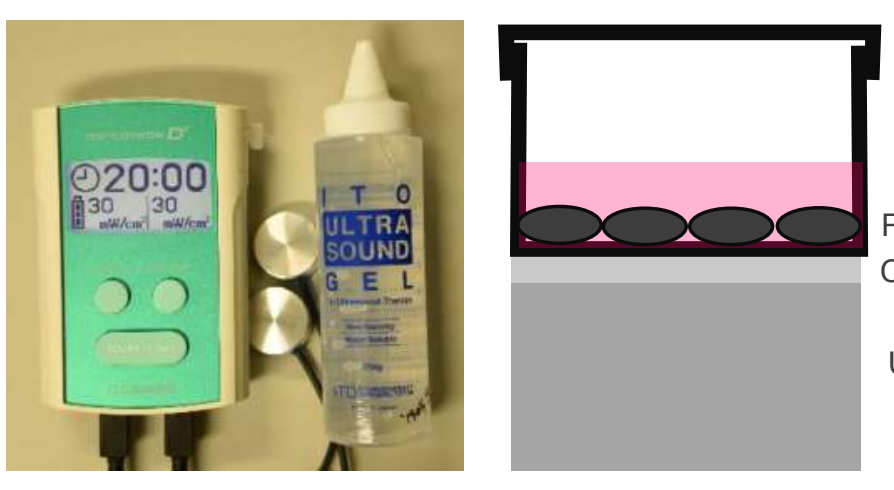

Figure 3. Low-intensity pulsed ultrasound application

- For cell proliferation, $5 \times 10^{3}$ cells were seeded into a 96-well culture plates. CCK-8 and Presto Blue assay were analyzed.

- Osteogenic differentiation was determined by alkaline phosphatase (ALP) staining, ALP activity assay, alizarin red staining and RT-real-time-PCR of osteogenic marker genes.
Results

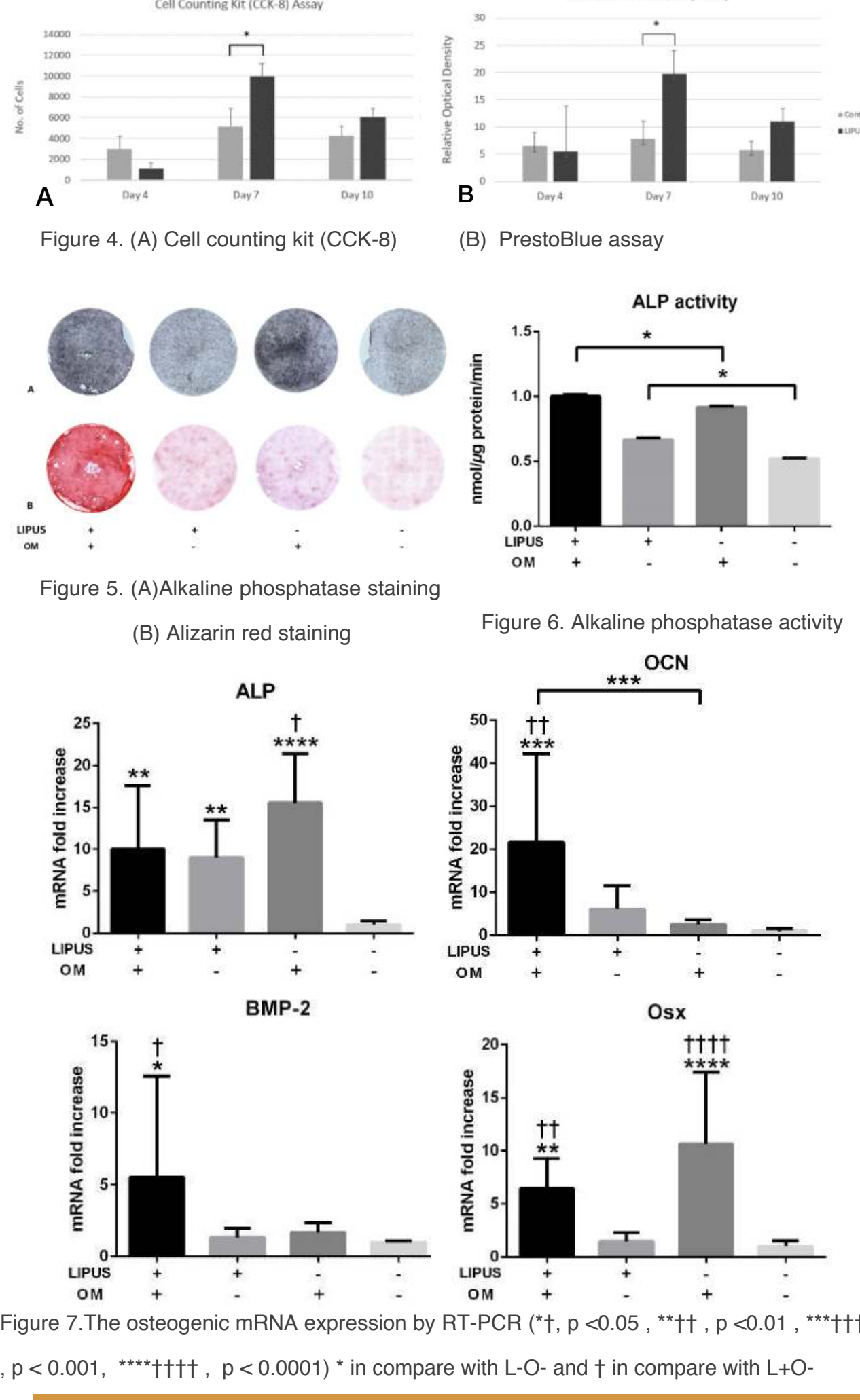

\section{Conclusion}

- LIPUS can significantly enhance PDCs cell proliferation after 7 days treatment. This indicated that LIPUS may have a positive effect on bone healing by enhancing periosteal cell proliferation.

$\mathrm{L}+\mathrm{O}+$ group showed more mineralized nodule formation and osteocalcin mRNA expression.

- $\mathrm{L}+\mathrm{O}+$ group increased BMP-2 mRNA expression and stimulated murine periosteal derived cell differentiation.

\section{References}

Harrison, A., Lin, S., Pounder, N., \& Mikuni-Takagaki, Y. (2016). Mode \& mechanism of low intensity pulsed ultrasound (LIPUS) in fracture repair. Ultrasonics, 70, 45-52.

. Kim, Y. K., Nakata, H., Yamamoto, M., Miyasaka, M., Kasugai, S., \& Kuroda, S. (2016). Osteogenic Potential of Mouse Periosteum-Derived Cells Sorted for CD90 In Vitro and In Vivo. Stem cells translational medicine, 5(2), 227-234.

Miyasaka, M., Nakata, H., Hao, J., Kim, Y. K., Kasugai, S., \& Kuroda, S. (2015). Low-intensity pulsed ultrasound stimulation enhances heat-shock protein 90 and mineralized nodule formation in mouse calvaria-derived osteoblasts. Tissue Engineering Part A, 21(23-24), 2829-2839. 